\title{
Leiomioma de uretra femenina, aportación de un caso clínico y revisión de la literatura
}

\section{Leiomyoma of the female urethra, a case and review of the literature}

\section{Sr. Director:}

Se presenta el caso de una mujer de 23 años de edad, sin antecedentes personales de interés, que acude a consulta de urología con motivo de disuria e infecciones de orina (cistitis) de repetición con sin respuesta al tratamiento médico mediante antibióticos. Junto con ello, refiere la existencia de una lesión excreciente localizada a nivel de meato uretral, de 4 meses de evolución, no dolorosa, firme y que se ha ido incrementando en tamaño en las últimas 4 semanas.

La exploración física revela una lesión exofítica, de color blanco-rosado, redondeada, de bordes bien limitados, firme y no adherida a planos profundos y unida a uretra distal, sin llegar a protuir por el meato uretral.

Ante la sospecha clínica de leiomioma de uretra se decide intervención quirúrgica programada.

Durante la intervención quirúrgica se evidencia la existencia de una lesión de color blanquecino, ovalada, de $1,5 \mathrm{~cm}$ de diámetro, base pediculada y localizada en uretra distal. Se realiza exéresis simple de la lesión.

La anatomía patológica revela una lesión bien delimitada, constituida por haces de células fusiformes, ausencia de mitosis, inexistencia de atipia y buena diferenciación celular. El examen inmunohistoquímico resultó positivo para actina.

Tras estos hallazgos se confirma el diagnóstico histopatológico de leiomioma uretral.

El leiomioma uterino es la patología tumoral más frecuente en la mujer; sin embargo, el leiomioma uretral es una patología rara ${ }^{1-10}$.

Descrito por primera vez por Buttner en 1984, existen 110 casos de estos tumores en la literatura inglesa encontrados por Bartolozzi et $\mathrm{al}^{10}$.

Este tumor suele devenir durante el periodo fértil femenino (desde la menarquia a la menopausia), la media de edad de aparición del esta patología esta en torno a 41 años de edad ${ }^{2,3}$.

Hoy en día su origen sigue siendo desconocido ${ }^{2,3}$. Se sabe que las hormonas ováricas favorecen su desarrollo, sin embargo es complicado saber cómo estas hormonas afectan exactamente el desarrollo del tumor, ya que existen casos documentados en pacientes posmenopáusicas ${ }^{9}$.

Las hormonas ováricas actúan a través de sus receptores específicos localizados intranucleo celular. Se encuentran en tejidos de órganos reproductores, tracto urinario inferior y sistema cardiovascular, mientras que otros tejidos del organismo están influenciados por estas hormonas sin evidencia de expresión de estos receptores.

De hecho, el tratamiento hormonal ha sido admitido como alivio sintomático del leiomioma por todos lo autores ${ }^{1-10}$.

Parece ser que los progestágenos son los principales responsables del desarrollo de este tumor (más que los estrógenos) como sugieren los hallazgos en biopsias de leiomioma durante el curso de la enfermedad. Kesari et al publicaron el primer reporte donde se documentaba la existencia de receptores estrogenitos positivos. En la mayoría de casos, la inmunohistoquimia revela la existencia de receptores de hormonas ováricas tipo ER $\alpha, E R \beta$ y PR en el núcleo de las células tumorales. Estos hallazgos, y el que un aumento de hormonas ováricas resultan en un aumento del ratio de mitosis tisular del leiomioma, apoyan la teoría de que estas hormonas sean promotoras del crecimiento tumoral ${ }^{9}$.

Los leiomiomas pueden surgir en cualquier lugar del tracto urinario, siendo el riñón y la vejiga (por este orden) los lugares más frecuentes ${ }^{4}$. La región más frecuentemente afecta a nivel uretral suele ser la uretra proximal, con una tamaño medio de $3,7 \mathrm{~cm}$ de diámetro según los datos recopilados por Bartolozzi et $\mathrm{al}^{10}$.

Pueden presentarse desde asintomáticos hasta aparecer en el contexto de un cuadro de $\mathrm{RAO}^{1-10}$, pasando por síntomas como dispareunia, hematuria, LOE uretral, ITU y síntomas irritativos (los 2 últimos son los mas frecuentes según la serie de Fry et al y Moopan et al) ${ }^{5,6}$.

El diagnóstico de esta patología se fundamenta en la historia clínica, la exploración física (masa haciendo impronta sobre la luz uretral, visible en leiomiomas localizados en uretra distal) y técnicas por imagen, de todos modos el diagnóstico definitivo se realiza mediante el examen histopatológico.

Pruebas con contraste radiológico, como la cistouretrografía retrógrada o la cistografía miccional permiten identificar defectos de repleción en estos pacientes. La uretrocistoscopia permite identificar estas lesiones bajo visión directa y la toma de muestra para biopsia.

Mediante un estudio ecográfico se permite identificar lesiones hipoecogénicas, bien delimitadas y homogéneas ${ }^{7}$.

En un trabajo publicado por Pavlica ${ }^{9}$ se realza la importancia del estudio por imagen en esta patología, y como se puede identificar la naturaleza benigna de la lesión mediante examen ecográfico transvaginal y RMN.

Las pacientes que presentan esta patología suelen tener clínica de ITU de repetición, esto se debe a la existencia de una obstrucción en el flujo de salida de orina y un remanso de orina que queda en el interior del tracto urinario. En el trabajo de Goto et al, se certifica la existencia de esta obstrucción al flujo mediante estudio urodinámico. Las pacientes con leiomioma de uretra presentan de alta presión del detrusor y un flujo máximo bajo con un residuo de orina posvaciado significativo en voluntarios vaciamiento y una resistencia uretral elevada. El estudio urodinámico tras la corrección quirúrgica revela una normalización en la presión del detrusor del flujo máximo y asuencia de residuo postmicción, traduciéndose clínicamente en una mejora de su dificultad miccional y dispareunia. 
En el diagnostico diferencial de esta patología se debe de incluir: carúncula uretral, leiomioma vesical extravesical, plasmocitoma y linfoma, y debe de diferenciarse del leiomiosarcoma (caracterizado por la atipa celular, el pleomorfismo y la elevada tasa de mitosis existente en el examen patológico de la lesión).

El tratamiento mas adecuado para esta patología es la escisión local ${ }^{1-10}$, durante la cirugía la mucosa uretral puede ser lesionada siendo necesaria su reparación (posible aparición de estenosis de uretra o IUE como complicaciones), si el leiomioma se libera sin necesidad de disrupción de la mucosa uretral, lo mas probable es que no proceda del músculo liso circular de la uretra.

Tras el tratamiento no suele existir recurrencia de la enfermedad ni malignización, solo existe un caso reportado de enfermedad recurrente, en una paciente con un tumor de mas de $8 \mathrm{~cm}$ de diámetro.

B I B L I O G R A F Í A

1. Goldman HB, McAchran SE, MacLennan GT. Leiomyoma of the urethra and bladder. J Urol. 2007;177:1890. No abstract available.

2. Chong KM, Chuang J, Tsai YL, Hwang JL. A rapidly growing paraurethral myoma with profuse bleeding from a mucosal vessel: report of a case. Gynecol Obstet Invest. 2006;61:87-9.
3. Ozel B, Ballard C. Urethral and paraurethral leiomyomas in the female patient. Int Urogynecol J Pelvic Floor Dysfunct. 2006;17:93-5 Epub 2005 May 19.

4. Diószeghy G, Kiss A, Kondás J. Leiomyoma of the female urethra. Int Urol Nephrol. 1998;30:603-7.

5. Fry M, Wheeler Jr JS, Mata JA, Culkin DJ, St Martin E, Venable DD. Leiomyoma of the female urethra. J Urol. 1988;140:613-4.

6. Mooppan MM, Kim H, Wax SH. Leiomyoma of the female urethra. J Urol. 1979;121:371-2.

7. Rivière $P$, Bodin R, Bernard G, Deligne E, Peyromaure M, Ponties JE. Leiomyoma of the female urethra. Prog Urol. 2004; 14:1196-8, discussion 1198.

8. Leidinger RJ, Das S. Leiomyoma of the female urethra. A report of two cases. J Reprod Med. 1995;40:229-31.

9. Pavlica P, Bartolone A, Gaudiano C, Barozzi L. Female paraurethral leiomyoma: ultrasonographic and magnetic resonance imaging findings. Acta Radiol. 2004;45:796.

10. Bortolozzi G, Grasso A, Zasso B. Leiomyoma of the female urethra. A case report and review. Clin Exp Obstet Gynecol. 1995;22:169-71.

D. Gallego Vilar*, I. José Povo Martin, J. Miralles Aguado, V. Gimeno Argente, M. Bosquet Sanz y J. Gallego Gomez

Servicio de Urología, Hospital General de Castellón, Castellón de la Plana, España

*Autor para correspondencia.

Correo electrónico: dagalvi@hotmail.com (D. Gallego Vilar). 